Revista Geográfica Digital. IGUNNE. Facultad de Humanidades. UNNE.

Año 14. № 27. Enero - Junio 2017. ISSN 1668-5180 Resistencia, Chaco

\title{
USO DEL SUELO CON FINES URBANOS EN LAS TERRAZAS Y ÁREAS MARGINALES DE LOS RÍOS PARAGUAY - PARANÁ
}

\author{
Jorge Alfredo Alberto \\ Directora: Doctora Ana María Foschiatti \\ Coodirectora: Profesora María Emilia Pérez
}

\section{- Resumen -}

El paisaje resulta de procesos en continua interacción en el tiempo y en el espacio que quedan reflejados en los usos del suelo. De allí que el objeto de estudio de este trabajo se refiere a las franjas próximas a centros urbanos, las que se encuentran sometidas al constante empuje del crecimiento de la ciudad a causa de la función residencial y de diferentes actividades complementarias (servicios e infraestructuras), que dan lugar a un espacio dinámico y complejo, ya que el intenso proceso de urbanización genera un sector especulativo que se apropia de tierras con renta agrícola para obtener renta urbana. Este "territorio de borde" está sometido a procesos económicos relacionados con la valorización capitalista del espacio, como consecuencia de la incorporación real o potencial de nuevas tierras a la ciudad.

En el área seleccionada para su estudio, se observa que el crecimiento urbano implica la incorporación de nuevos territorios rurales en el complejo sistema que comprende un espacio en mutación, como lo es la ciudad y sus áreas de transición, sin tener en cuenta para ello las relaciones existentes entre las demandas que incorporan estas propuestas y la dinámica de los condicionantes naturales, conjuntamente con la dinámica propia de las actividades tradicionales previas a este proceso (agrícolas, ganaderas), lo cual complejiza las problemáticas ambientales derivadas de las transformaciones del espacio involucrado.

El crecimiento de este espacio de transición del AMGR presenta cuatro formas espaciales básicas reconocibles de ocupación con fines urbanos, que son: desarrollo continuo de baja densidad, desarrollo en faja, desarrollo discontinuo o en "salto de rana" y desarrollo disperso extraurbano. El efecto de este fenómeno es el aumento de sistemas de infraestructura viaria y de movilidad intraurbana, que acentúa el crecimiento de la mancha urbana concomitante con bolsas de marginalidad, vacíos urbanos y pobreza, a lo que se suman espacios urbanizados con un alto grado de vulnerabilidad hídrica.

Esta aproximación de análisis sobre la ocupación de lo urbano sobre lo rural, con especial énfasis en los espacios de transición, permitió deducir el comportamiento de la situación de la tierra, verificar la organización de la misma y los problemas que afectan al área en cuestión.

Palabras Claves: Crecimiento urbano, espacios de transición urbano rurales, condiciones hidrogeomorfológicas, tendencia natural, acción antrópica, uso del suelo, problemáticas, diagnóstico y gestión.

Publicado en formato digital: Dr. Prof. Jorge Alfredo Alberto. USO DEL SUELO CON FINES URBANOS EN LAS TERRAZAS Y ÁREAS MARGINALES DE LOS RÍOS PARAGUAY - PARANÁ. RESÚMENES DE TESIS. Revista Geográfica Digital. IGUNNE. Facultad de Humanidades. UNNE. Año 14. № 27. Enero - Junio 2017. ISSN 1668-5180 Resistencia, Chaco

En: http://hum.unne.edu.ar/revistas/geoweb/default.htm 
Revista Geográfica Digital. IGUNNE. Facultad de Humanidades. UNNE.

Año 14. № 27. Enero - Junio 2017. ISSN 1668-5180 Resistencia, Chaco

\title{
USO DEL SUELO CON FINES URBANOS EN LAS TERRAZAS Y ÁREAS \\ MARGINALES DE LOS RÍOS PARAGUAY - PARANÁ
}

\author{
Jorge Alfredo Alberto \\ Directora: Doctora Ana María Foschiatti \\ Coodirectora: Profesora María Emilia Pérez \\ - Resumen extendido -
}

\section{Introducción}

La presente tesis tiene como meta estudiar desde la Geografía algunas características distintivas del crecimiento urbano del Área Metropolitana del Gran Resistencia, poniendo especial énfasis en el desarrollo que se produce en los espacios de transición urbanos - rurales de dicho aglomerado.

En el mismo se tienen en cuenta el fenómeno de la expansión urbana asociados con el desarrollo de la mancha urbana y la fragmentación territorial, a los que se suman los condicionantes hidrogeomorfológicos y la tendencia natural del sitio. Estos elementos, junto con la dinámica del sistema, definen la traza y crecimiento de la ciudad y su comportamiento da lugar a problemáticas en relación con la degradación de los entornos y la vulnerabilidad de su población.

En este aspecto, la tendencia natural junto con los procesos antrópicos actúan, en conjunto, en la configuración de áreas con fisonomías particulares. Los resultados de estos procesos e interacciones, ya sean de orden natural o antrópico configuran en el espacio un mosaico de fisonomías denominados paisajes.

La noción de "paisaje" aporta la dimensión socio-cultural a los problemas de ordenación del territorio, por lo que podría enriquecer las propuestas de gestión territorial con el conocimiento más profundo de la relación entre los procesos naturales, sociales, económicos y culturales, facilitando la comprensión de la sociedad en general con respecto de las transformaciones causadas por estos procesos.

Para ello, el marco metodológico para el estudio geográfico del tema en cuestión se basa en el sistema GTP (geosistema, territorio, paisaje) presentado por Claude y Georges Bertrand (1968), que nos permite entender los procesos y características principales del paisaje en su dimensión geográfica

Valiéndose de este enfoque "geográfico y sistémico" que puede significar el punto de partida de nuevas interpretaciones, el trabajo de tesis aportará un ensayo conformado por un

Publicado en formato digital: Dr. Prof. Jorge Alfredo Alberto. USO DEL SUELO CON FINES URBANOS EN LAS TERRAZAS Y ÁREAS MARGINALES DE LOS RÍOS PARAGUAY - PARANÁ. RESÚMENES DE TESIS. Revista Geográfica Digital. IGUNNE. Facultad de Humanidades. UNNE. Año 14. № 27. Enero - Junio 2017. ISSN 1668-5180 Resistencia, Chaco

En: http://hum.unne.edu.ar/revistas/geoweb/default.htm 
conjunto ordenado de representaciones gráficas y cartográficas, con su correspondiente vertido de conceptos.

\section{Métodos y Técnicas}

Para el desarrollo del trabajo se aplicó un método para el estudio del paisaje apoyado en una concepción geográfica, siguiendo los principios de la geografía (localización, causalidad, conexión y evolución) y focalizada en la visión sistémica para concluir en la síntesis geográfica.

Este enfoque respondió a la necesidad de detectar y caracterizar los principales tipos de paisajes resultantes de procesos naturales y antrópicos en el AMGR y sus alrededores, centrados en la expansión urbana de dicha metrópolis sobre espacios rurales; valiéndose para ello de bases cartográficas, de síntesis e históricas, complementadas con fotografías aéreas e imágenes satelitales. Estos recursos estuvieron orientados al uso de herramientas informáticas que permitieron analizar y evaluar las diferentes variables que intervienen en dicho proceso.

\section{La urbanización en el contexto regional}

Los aspectos más importantes del crecimiento urbano de las ciudades del NEA durante estos últimos años estuvieron asociados al impacto producido a principios de la década pasada por el proceso de privatización de las empresas del estado, la desregulación y apertura de las actividades económicas y los efectos iniciales de la estabilización de la economía y la expansión del crédito, que dinamizó y modernizó algunas actividades comerciales y de servicios vinculadas a los sectores financieros, energéticos, de comunicación y de la construcción.

El resultado sobre el espacio urbano de estos procesos trajo aparejado, por una parte, la renovación de la imagen urbana del centro de estas ciudades y de algunas áreas revalorizadas, como producto del gasto público en obras de infraestructura y la inversión privada en el sector comercial de servicios e inmobiliario, mientras que, por otra parte, la expansión de grandes áreas residenciales de baja calidad ambiental y servicios deficientes como producto de la acción autogestionaria de los sectores populares y la política habitacional pública, que han contribuido a definir un espacio urbano de contrastes y fragmentación social.

Sobre este proceso tuvo una incidencia importante la segregación urbana producida por la implantación de grandes obras públicas de infraestructura en las principales ciudades de la región, que contribuyeron a redefinir áreas socialmente más homogéneas hacia su interior y dispares entre ellas, en términos jerárquicos, como así también, la baja calidad del espacio público de estas áreas promovida por las políticas públicas, que no han contribuido a la integración social de la población.

Publicado en formato digital: Dr. Prof. Jorge Alfredo Alberto. USO DEL SUELO CON FINES URBANOS EN LAS TERRAZAS Y ÁREAS MARGINALES DE LOS RÍOS PARAGUAY - PARANÁ. RESÚMENES DE TESIS. Revista Geográfica Digital. IGUNNE. Facultad de Humanidades. UNNE. Año 14. № 27. Enero - Junio 2017. ISSN 1668-5180 Resistencia, Chaco

En: http://hum.unne.edu.ar/revistas/geoweb/default.htm 
Revista Geográfica Digital. IGUNNE. Facultad de Humanidades. UNNE. Año 14. № 27. Enero - Junio 2017. ISSN 1668-5180 Resistencia, Chaco

\section{Condiciones hidrogeomorfológicas del sitio}

Las características morfométricas del sitio del AMGR. y zonas aledañas son el resultado de la dinámica del río Paraná que se comporta como un gran regulador hídrico y topográfico del espacio sujeto a estudio debido a la magnitud de su cuenca y el caudal que transporta, lo que genera innumerables formas fluviales (actuales o abandonadas) que inciden en el relieve generando irregularidades topográficas. Las acción antrópica sobre este espacio natural, se han hecho sentir desde los albores de la ocupación, tanto por los usos de suelo agrícola de los albardones como por la explotación forestal. La expansión del área edificada, los servicios e infraestructuras como el trazado de vías de comunicación, se dio en todos sentidos, pero en especial, con miras a la conexión del puerto con las áreas de producción locales y con las áreas más lejanas del territorio.

En consecuencia se modificó así la fisonomía del área de influencia hidrológica del río Negro y de su propia faja de divagación, no solo por la supresión de elementos sino por las alteraciones de los que quedaron, fenómeno que se repite en la actualidad en las riberas del río Tragadero y planicies encerradas, próximas a Colonia Benítez. Esta situación de relleno de las lagunas, de su fragmentación o de su aislamiento del curso principal, no sólo incide en la depresión al modificarse la primitiva morfología, sino que se altera el comportamiento hidrológico que a ella se asocia.

Como es el caso del AMGR., la mayoría de los "problemas ambientales" dependen de desajustes "iniciales" en la selección o utilización del sitio original. En ocasiones, la transformación del espacio natural inicial por usos intensivos, supera condiciones naturales generando un cierto "colapso" de tal sitio. La transformación del entorno natural por efectos de la urbanización, constituye una de las formas más intensivas de modificación de dicho entorno, desarrollándose en ciertos casos, situaciones ambientales extremadamente críticas e irreversibles.

\section{El AMGR. como organizadora de la dinámica de desarrollo urbano}

El crecimiento del AMGR presenta cuatro formas espaciales básicas reconocibles de ocupación con fines urbanos, que son; desarrollo continúo en baja densidad, desarrollo en faja, desarrollo discontinuo o en "salto de rana", y desarrollo disperso extraurbano. El efecto de éste fenómeno es el aumento de sistemas de infraestructura viaria y de movilidad intraurbana que acentúan el crecimiento de la mancha urbana concomitante con bolsas de marginalidad, vacíos urbanos y pobreza, a lo que se suman espacios urbanizados con un alto grado de vulnerabilidad hídrica.

La ausencia de planeamiento en las periferias urbanas es muy visible. Ahí está la realidad: una extrema degradación ambiental, una acentuada desarticulación funcional urbana entre las colonias periféricas y la ciudad, un uso del suelo irracional, una dispersión e

Publicado en formato digital: Dr. Prof. Jorge Alfredo Alberto. USO DEL SUELO CON FINES URBANOS EN LAS TERRAZAS Y ÁREAS MARGINALES DE LOS RÍOS PARAGUAY - PARANÁ. RESÚMENES DE TESIS. Revista Geográfica Digital. IGUNNE. Facultad de Humanidades. UNNE. Año 14. № 27. Enero - Junio 2017. ISSN 1668-5180 Resistencia, Chaco

En: http://hum.unne.edu.ar/revistas/geoweb/default.htm 
insuficiencia de equipamiento y servicios, una masa humana desintegrada socialmente que genera problemas de desadaptación y falta de sentido de pertenencia al lugar en que viven por la ausencia de identidad con el mismo.

\section{Condiciones y dinámicas de un espacio ruderal sujeto a la presión de la expansión urbana}

Entre las características más destacables del sitio que comprende el área sujeta a estudio se pueden detallar que es una planicie aluvial compleja donde se observa la presencia de terrenos elevados de baja amplitud que están ocupados por relictos de leñosas cuyas poblaciones son tolerantes a la acción de las inundaciones y sequías moderadamente prolongadas. Estos se encuentran intercalados entre terrenos bajos cuyo escurrimiento lento se produce de manera laminar con un gran desarrollo areal donde la permanencia de agua está condicionada por el efecto de frenado que generan los obstáculos topográficos y biológicos, y que se acentúan con los antrópicos.

En esta interfase urbano - rural estudiada se observa que la coexistencia de ambos usos es contradictoria, ya que en la misma se dificulta la convivencia de su población en relación a intereses disímiles, pero se tolera la competencia entre el desarrollo urbano y los usos rurales, recibiendo en consecuencia todo tipo de población: población urbana en búsqueda de menores costos de vida o mejores condiciones ambientales y de vida, población rural en búsqueda de nuevas oportunidades de trabajo por la reducción y degradación de sus tierras, y la implantación de actividades de servicios propios de la vida urbana (oficios, domesticas, etc.), que en su conjunto responden a las dinámicas regionales y nacionales desde la pequeña a la gran escala.

\section{Dinámica y configuración de los usos del suelo de un espacio ruderal sujeto a la presión de la expansión urbana}

Lo planteado con anterioridad evidencia la baja integración entre lo natural y social, ya que no se tienen en cuenta simultáneamente los diversos tipos de actores sociales, los objetivos y metas oficiales - sectoriales, la incidencia que tiene el efecto de intervenciones externas para obtener beneficios a corto plazo, las políticas de desarrollo y sus cambios rápidos, la repartición de los beneficios del uso de los recursos, etc.

Esta problemáticas, en su conjunto, dan lugar a la pérdida de atractivo económico de las producciones primarias (agrícola, ganadera, hortícola y forestal) en la zona, generando una transformación en la valoración del uso del suelo, ya que este deja de tener un valor especulativo desde el punto de vista productivo (aptitud agrícola, receptividad ganadera, etc.) para adquirir un valor especulativo de tipo inmobiliario (distancia a la ruta y centros poblados, servicios, etc.), que deriva en la acentuación de procesos de urbanización a partir de loteos y edificaciones.

Publicado en formato digital: Dr. Prof. Jorge Alfredo Alberto. USO DEL SUELO CON FINES URBANOS EN LAS TERRAZAS Y ÁREAS MARGINALES DE LOS RÍOS PARAGUAY - PARANÁ. RESÚMENES DE TESIS. Revista Geográfica Digital. IGUNNE. Facultad de Humanidades. UNNE. Año 14. № 27. Enero - Junio 2017. ISSN 1668-5180 Resistencia, Chaco

En: http://hum.unne.edu.ar/revistas/geoweb/default.htm 
Estos procesos, organizados en grupos, incidirán en la dinámica y el comportamiento de la evolución del paisaje por medio de una secuencia lógica de cambios de propiedades que responderían, en su conjunto a la acción antrópica sobre el área de estudio, que de no tomarse las medidas preventivas pertinentes se acentuarían, dando lugar a problemáticas aún más complejas que las diagnosticadas y analizadas en este trabajo, por lo tanto más difíciles de paliar y solucionar. Se observa que el proceso de expansión urbana sobre el espacio sujeto a estudio:

- contribuye a un debilitamiento de los equipamientos y servicios públicos.

- disminuye el mantenimiento efectivo de las infraestructuras existentes.

- incrementa los costos sociales de transporte.

- consume más recursos que otros patrones de desarrollo urbano.

- limita a gran parte de la población local al acceso a puestos de trabajo en el AMGR.

- incrementa los gastos en el tiempo de transporte.

- degrada la calidad del aire y del agua.

- supone una alteración o destrucción permanente de los hábitats naturales.

\section{Comportamiento tendencial y deseado de la dinámica de los usos del suelo de un espacio ruderal sujeto a la presión de la expansión urbana}

Como se ha mencionado en apartados anteriores, en la identificación de las problemáticas se parte de un enfoque integrado y sistémico del paisaje, con lo pretensión de brindar lineamientos para el ordenamiento ambiental que el sistema territorial debe alcanzar.

Para dicho planteo se trabajó sobre dos grandes sistemas, el natural y el antrópico; en el primero se tuvo en cuenta solamente aspectos físicos y biológicos que caracterizan el comportamiento del área de estudio, sin por ello pasar por alto la acción humana sobre los mismos. En cambio, el sistema antrópico se organizó a partir de cuatros aspectos dinámicos e interrelacionados entre sí: el productivo (explotación forestal, ganadera, agrícola, hortícola e industrial), el inmobiliario, la población y las infraestructuras y servicios.

En relación al sistema natural, tanto el aspecto físico como el biológico presentan una fuerte degradación del medio ambiente, resultado de la acentuación de procesos naturales (erosión, colmatación, inundación) y la alteración de las formaciones vegetales originales a través de la acción antrópica (dilapidación, sobreexplotación, avance de invasoras, extinción)

Con respecto al sistema antrópico, en el aspecto productivo, que implica las actividades primarias y de transformación, la mayor parte de los problemas identificados están relacionados con la degradación del medio por sobreexplotación, erosión, compactación, contaminación y enfermedades, sumados a la escasa rentabilidad económica productiva del mismo por falta de coordinación y complementación de la explotación a nivel predio, a lo que se añade la escasa transformación, preservación, promoción y comercialización de los productos obtenidos.

Publicado en formato digital: Dr. Prof. Jorge Alfredo Alberto. USO DEL SUELO CON FINES URBANOS EN LAS TERRAZAS Y ÁREAS MARGINALES DE LOS RÍOS PARAGUAY - PARANÁ. RESÚMENES DE TESIS. Revista Geográfica Digital. IGUNNE. Facultad de Humanidades. UNNE. Año 14. № 27. Enero - Junio 2017. ISSN 1668-5180 Resistencia, Chaco

En: http://hum.unne.edu.ar/revistas/geoweb/default.htm 
En el aspecto inmobiliario se hace referencia a los fines de ocupación del suelo. En el mismo, se observa una alteración de las cualidades espaciales por una ocupación anárquica, resultado de una acentuada especulación inmobiliaria, que responde, en primer lugar, a un acelerado crecimiento demográfico y a una densificación urbana por procesos migratorios de ámbitos rurales cercanos y de la ciudad de Resistencia, y en segundo lugar, por su proximidad en distancia y tiempo a esta última, la cual, en las décadas del 80 y 90 ha tenido un rápido crecimiento del espacio urbanizado, acentuándose dicho proceso en estos últimos 15 años.

Esta problemática deriva en un loteo de tierras productivas, artificialización del paisaje con praderas y bosques monoespecíficos de especies endógenas y exógenas indistintamente, deficiencia de servicios e infraestructuras existentes, situación que se profundiza por la ausencia de un proyecto de planificación de expansión urbana y por la carencia de políticas fiscales acordes al uso del suelo.

En el aspecto demográfico se visualiza un crecimiento acelerado de la población, la que se concentra en núcleos ubicados en los parajes Tres Horqueta, y El Tropezón y en el pueblo de Colonia Benitez. Gran parte de esta proviene de ámbitos rurales aledaños, donde las actividades agrícolas se encuentran en una situación de crisis y recesión; aunque en los últimos años, como se comentó más arriba, se observa una marcada tendencia de población proveniente del AMGR en busca de esparcimiento o con fines residenciales, proceso acompañado por un movimiento pendular por cuestiones laborales.

En el caso de la población local, se observa un número importante de personas desocupadas con demandas insatisfechas de viviendas y servicio básicos, lo cual genera asentamientos marginales, bajo condiciones de ilegalidad, en áreas con riesgo de inundación, lo que pone en peligro la integridad física de los mismos, situación que profundiza la presencia de bolsones de pobreza, incrementando hechos delictivos y de inseguridad, tanto en los bienes como en las personas en general.

En relación a la segunda, el posible incremento de los espacios urbanizados a través de viviendas $y$, el creciente interés en adquisición inmobiliaria con un fuerte loteo $y$ artificialización de tierras productivas sin un plan rector que las oriente, advierten para un futuro próximo el carácter urbano de la zona con una densidad de saturación de suelo muy importante, donde la capacidad de contención de los servicios e infraestructuras se verán superadas, tornándose deficientes. Esta situación, junto a la falta de coordinación de los actores locales, profundizaría la especulación inmobiliaria alterando las cualidades espaciales y la forma de vida de sus pobladores, a lo que se sumaría un incremento de la confrontación entre vecinos por diferencias de intereses y de costumbres.

En el aspecto de infraestructuras y servicios, las problemáticas observadas son el resultado del marcado crecimiento de la población y el acelerado aumento de las superficies urbanizadas y construidas, complementadas con procesos de ocupación de espacios rurales con fines productivos. Se observa que en ámbitos rurales éstas no respetan la tendencia

Publicado en formato digital: Dr. Prof. Jorge Alfredo Alberto. USO DEL SUELO CON FINES URBANOS EN LAS TERRAZAS Y ÁREAS MARGINALES DE LOS RÍOS PARAGUAY - PARANÁ. RESÚMENES DE TESIS. Revista Geográfica Digital. IGUNNE. Facultad de Humanidades. UNNE. Año 14. № 27. Enero - Junio 2017. ISSN 1668-5180 Resistencia, Chaco

En: http://hum.unne.edu.ar/revistas/geoweb/default.htm 
Revista Geográfica Digital. IGUNNE. Facultad de Humanidades. UNNE.

Año 14. № 27. Enero - Junio 2017. ISSN 1668-5180 Resistencia, Chaco

natural en la mayoría de los casos, por ejemplo el trazado de caminos y alcantarillados deficientes que limitan el escurrimiento del exceso hídrico. En cambio, en ámbitos urbanos se nota la demanda insatisfecha de viviendas de planes sociales, la deficiencia en el abastecimiento de agua potable, la limitada capacidad de contención del sistema educativo y sanitario, y finalmente la carencia de servicios básicos de cloacas, situación que demuestra una notoria pérdida en la calidad de vida de los pobladores.

Además se percibe un descuido de los patrimonios culturales y naturales, por aislamiento e indiferencia a los intereses de la comunidad por parte de los principales actores sociales, o bien, de población inserta proveniente de otras zonas que no se sienten representados por las mismas pautas o ideales.

Definitivamente la falta de cohesión y coordinación, a nivel local, para el mantenimiento y ampliación de infraestructuras y servicios impactan de manera negativa en los sistemas estructurales del equipamiento comunitario, acentuando la crisis en la calidad vida de la población.

Finalmente, teniendo en cuenta lo tratado hasta aquí, se pueden proponer a través de árboles de objetivos posibles oportunidades para superar los problemas, es decir a partir de las situaciones conflictivas o de desequilibrio detectadas se formulan una serie de condiciones o situaciones esperadas en el futuro, que son deseadas y realizables en la práctica.

En síntesis, todos los problemas identificados se resumen en cuatro problemas claves, que se detallan a continuación:

1. Degradación del medio natural original en su totalidad.

2. Sistemas productivos ineficientes en relación a rentabilidad y sostenibilidad.

3. Alteración de las cualidades espaciales.

4. Pérdida de la calidad de vida.

A partir de los problemas claves, se han definido los siguientes objetivos tendientes a su solución:

1. Paliar y/o disminuir la degradación del medio natural.

2. Fomentar iniciativas para mejorar la eficiencia de los sistemas productivos.

3. Disminuir la alteración de las cualidades espaciales.

4. Paliar o disminuir la pérdida de la calidad de vida.

\section{Conclusión}

En el área seleccionada para su estudio, se observa que el crecimiento urbano implica la incorporación de nuevos territorios rurales en el complejo sistema que comprende un espacio en mutación como lo es la ciudad y sus áreas de transición, sin tener en cuenta para ello las relaciones existentes entre las demandas que incorporan estas propuestas y la dinámica de los condicionantes naturales junto con la dinámica propia de las actividades

Publicado en formato digital: Dr. Prof. Jorge Alfredo Alberto. USO DEL SUELO CON FINES URBANOS EN LAS TERRAZAS Y ÁREAS MARGINALES DE LOS RÍOS PARAGUAY - PARANÁ. RESÚMENES DE TESIS. Revista Geográfica Digital. IGUNNE. Facultad de Humanidades. UNNE. Año 14. № 27. Enero - Junio 2017. ISSN 1668-5180 Resistencia, Chaco

En: http://hum.unne.edu.ar/revistas/geoweb/default.htm 
tradicionales previas a este proceso (agrícolas, ganaderas), lo cual complejiza las problemáticas ambientales derivadas de las transformaciones del espacio involucrado.

El crecimiento de este espacio de transición del AMGR presenta cuatro formas espaciales básicas reconocibles de ocupación con fines urbanos, que son; desarrollo continúo en baja densidad, desarrollo en faja, desarrollo discontinuo o en "salto de rana", y desarrollo disperso extraurbano:

- El desarrollo continuo en baja densidad, es el de mayor consumo de territorio en los márgenes del área metropolitana, transformándolo en suelo urbano. Este tipo de expansión se basa en la extensión sectorizada de las redes de infraestructura urbana como ser agua, electricidad, etc. (Villa Fabiana, Villa Camila, Monte Alto, La California, etc.)

- El desarrollo en faja se da en la línea de los grandes corredores de transporte que salen de las zonas centrales de la ciudad (en especial la ruta Nacional $n^{\circ} 11$ y la Autovía Nicolás Avellaneda) Las zonas adyacentes al corredor se desarrollan con usos urbanos, y las zonas alejadas al mismo mantienen su carácter rural. Con el tiempo esta tierra rural se convierte a urbana, en la medida que surjan corredores transversales de transporte o caminos vecinales.

- El desarrollo discontinuo o en "salto de rana" (Sprawl), son urbanizaciones discontinuas de los márgenes del área metropolitana o de conurbaciones en faja, como el caso de las urbanizaciones próximas a la localidad de Colonia Benítez (Las Marías, Chacras de Benítez, etc.) Este tipo de desarrollo es el que requiere de mayor inversión en servicios urbanos para poder materializarse.

- El desarrollo disperso extraurbano se asocia al concepto de expansión en vivienda de tipo parcelación, en entornos naturales (agrícolas, forestales, etc.), más allá de los suburbios de la ciudad. Este patrón de asentamiento ha sido también denominado "desarrollo en baja densidad extendido", y difiere significativamente de los patrones urbanos, de suburbios o rurales. Los mismos se encuentran en un acentuado desarrollo en el trayecto comprendido entre Margarita Belén y Colonia Benítez próximos a la ruta Nacional $n^{\circ} 11$ y el viejo camino entre estas dos localidades conocido como "La diagonal".

El producto resultante de los cuatro procesos de urbanización antedichos provoca impactos evidentes tanto en el AMGR como en el área seleccionada para estudio tales como: la pérdida de población de los espacios urbanos consolidados que genera para las administraciones de las ciudad disminución y evasión en la percepción de impuestos; el aumento en los gastos de la infraestructura viaria difícil de financiar con las bajas densidades; el consumo de tierras periurbanas destinadas a la producción, la subutilización del centro urbano, a lo que se suma la segregación residencial en relación a la diferenciación en cuanto a los tipos de servicios (compras, entretenimientos, educación, salud)

En este "perímetro", en constante aumento, conviven todas las posibilidades comentadas que traen como consecuencia las demandas de requisitos muy dispares, complejos y difíciles de satisfacer, dando lugar a una variedad de problemáticas sociales,

Publicado en formato digital: Dr. Prof. Jorge Alfredo Alberto. USO DEL SUELO CON FINES URBANOS EN LAS TERRAZAS Y ÁREAS MARGINALES DE LOS RÍOS PARAGUAY - PARANÁ. RESÚMENES DE TESIS. Revista Geográfica Digital. IGUNNE. Facultad de Humanidades. UNNE. Año 14. № 27. Enero - Junio 2017. ISSN 1668-5180 Resistencia, Chaco

En: http://hum.unne.edu.ar/revistas/geoweb/default.htm 
Revista Geográfica Digital. IGUNNE. Facultad de Humanidades. UNNE. Año 14. № 27. Enero - Junio 2017. ISSN 1668-5180 Resistencia, Chaco

legales, económicas y en su conjunto, ambientales. Como respuesta a la situación antedicha, los grupos humanos deben recurrir a los espacios periféricos y vacíos urbanos que pueden encontrarse en riesgo debido a que estos territorios, lejos de reunir cualidades de habitabilidad, son vulnerables por sus condiciones físico - naturales.

Esta aproximación de análisis sobre la ocupación de lo urbano sobre lo rural, con especial énfasis en los espacios de transición, permitió deducir el comportamiento de la situación de la tierra, verificar la organización de la misma y los problemas afectan al área en cuestión. Teniendo en cuenta los aspectos antedichos que inciden sobre el área de estudio surge la necesidad de profundizar e insistir en las líneas de acción futuras como:

- Estudiar las tecnologías posibles de aplicar sin pérdida de puestos de trabajo por expulsión.

- Desarrollar sistemas productivos novedosos, alternativos, complementarios y diversificados que exploten los recursos locales de manera sustentable.

- Definir y potenciar centros críticos de desarrollo económico local, poniendo el esfuerzo en identificar núcleos de urbanización que presenten un potencial desarrollo en apoyo de las actividades agropecuarias.

- Trabajar en el incremento del intercambio comercial entre centros poblados cercanos para que se conviertan, además, en centros de distribución de los productos agrarios.

- Paliar el acceso insuficiente o la mala distribución de tierras, ya sea por obstáculos políticos, sociales, inmobiliarios o económicos.

- Legislar para limitar la usurpación de tierras por medio de un sistema no sujeto a la escrituración usual de la propiedad privada, para ello se deberá determinar la propiedad de las parcelas y registrar el derecho de los individuos sobre los terrenos, a partir de normas que resulten accesibles a los grupos desfavorecidos y de bajos ingresos.

- Proponer medidas enérgicas para limitar la expansión urbana en las zonas agrícolas fértiles, conservándolas para la producción de alimentos y como espacios libres de contaminación.

- Construir la estructura que orienten el cambio de la actividad urbana y de la actividad rural, trabajando a favor de educar en una cultura acorde con el desarrollo sustentable.

\section{Bibliografía base consultada}

1. Alberto, Jorge A. (2005) "Problemáticas ambientales resultantes de la ocupación urbana en ambientes fluviolacustres. Estudio de caso: Área Metropolitana del Gran Resistencia (AMGR)”. En Revista Geográfica no 138. Instituto Panamericano de Geografía e Historia (IPGH) Mexico DF. Julio - diciembre 2005. pp. 109 - 127

2. Alberto, Jorge A. (2007) "Vulnerabilidad Ecológica y natural". En: Foschiatti, Ana Maria (Eds.) "Aportes conceptuales y empiricos de la vulnerabilidd global". Resistencia. EUDENE. UNNE. Resistencia. Argentina. pp. 117 - 158.

Publicado en formato digital: Dr. Prof. Jorge Alfredo Alberto. USO DEL SUELO CON FINES URBANOS EN LAS TERRAZAS Y ÁREAS MARGINALES DE LOS RÍOS PARAGUAY - PARANÁ. RESÚMENES DE TESIS. Revista Geográfica Digital. IGUNNE. Facultad de Humanidades. UNNE. Año 14. № 27. Enero - Junio 2017. ISSN 1668-5180 Resistencia, Chaco

En: http://hum.unne.edu.ar/revistas/geoweb/default.htm 
Revista Geográfica Digital. IGUNNE. Facultad de Humanidades. UNNE. Año 14. № 27. Enero - Junio 2017. ISSN 1668-5180 Resistencia, Chaco

3. Barnes, K., Morgan, J. (2002) "Sprawl development: its patterns, consequences, and measurement" Center for Geographic Information Sciences, Towson University. [en línea] Junio 2010: http://pages.towson.edu/morgan/files/Sprawl_Development.pdf

4. Barreto, M. A. y Ebel, G. A. (2013) "Crecimiento, distribución y composición de la problacion urbana y rural en el Chaco entre 1991 y 2010". Revista ADNea n 1. Vol. 1. Diciembre 2013, Facultad de Arquitectura y Urbanismo. UNNE. pp. 77 - 88.

5. Bertrand, G. (1968) "Paysage et geographie physique globale: esquisse méthodologique". Rev.Géograph. Pyrénées et du Sud-Ouest, 39(3):249-272, Toulouse.

6. Bolòs, I.; Capdevila, M. (1992) "Manual de Ciencia del Paisaje. Teoría, métodos y aplicaciones". Masson, Barcelona. $252 \mathrm{pp}$.

7. Bryant, C y Russwurn, L. Fuente: Bryant, C. R. ;Russwurn, L. H.,McLellan, A. G. (1982) "The city's countryside. Land and its management in the rural-urban fringe". Longman.- New York.- $249 \mathrm{pp}$.

8. Dollfus, O. (1976) "El espacio geográfico". Colección ¿Qué se? № 111, Ed. Oikos-Tau, Vilassar de Mar.

9. Foschiatti, A. M. (Eds.) (2008) "Aportes conceptuales y empiricos de la vulnerabilidd global". Resistencia. EUDENE. UNNE. Resistencia. Argentina. pp. 425.

10. García, R. (2006) "Sistemas complejos: conceptos, método y fundamentación epistemológica de la investigación interdisciplinaria". Editorial Gedisa. Barcelona.

11. Gaviria, M. (1985) "La competencia rural-urbana por el uso de la tierra", Agricultura y Sociedad, pp. 245-261

12. Ibarra Benlloch, P. (1993) "Una propuesta metodológica para el estudio del paisaje integrado". En revista Geographicalia 30. Revista del Departamento de Geografía y Ordenación del Territorio. Universidad de Zaragoza. Diciembre 1993. pp. 229-242

13. Mignone, A. M. (2004) "El proceso de urbanización en el Gran Resistencia, a principios del siglo XXI. El caso de los asentamientos espontáneos". Actas VI Encuentro Internacional Humboldt. Villa Carlos Paz., Córdoba. 13 a 17 de septiembre de 2004.

14. Pierre George (1985) "La acción del hombre y el medio geográfico". Traducción de Alexandre Montserrant. Ediciones Península, Historia, Ciencia, Sociedad no 61. Barcelona. España.

15. Pilar Serra, Y. (2003) "Resistencia: geomorfología del sitio, expansión urbana y afectación hídrica". XIII Encuentro Nacional de Profesores de Geografía. Agosto 2003.

16. Popolizio, E. (1982) "La geomorfología en los estudios ecológicos de la llanura". En revista. Geociencias XII. Publicación del Centro de Geociencias Aplicadas. Universidad Nacional del Nordeste. Resistencia, Chaco, Argentina.

17. Popolizio, E. (1989) "Algunos elementos geomorfológicos condicionantes de la organización espacial y las actividades del NEA". En revista Geociencias no XVII. Centro de Geociencias Aplicadas, UNNE. Resistencia.

Publicado en formato digital: Dr. Prof. Jorge Alfredo Alberto. USO DEL SUELO CON FINES URBANOS EN LAS TERRAZAS Y ÁREAS MARGINALES DE LOS RÍOS PARAGUAY - PARANÁ. RESÚMENES DE TESIS. Revista Geográfica Digital. IGUNNE. Facultad de Humanidades. UNNE. Año 14. № 27. Enero - Junio 2017. ISSN 1668-5180 Resistencia, Chaco

En: http://hum.unne.edu.ar/revistas/geoweb/default.htm 
Revista Geográfica Digital. IGUNNE. Facultad de Humanidades. UNNE.

Año 14. № 27. Enero - Junio 2017. ISSN 1668-5180 Resistencia, Chaco

\title{
LAND USE FOR URBAN PURPOSES ON THE TERRACES AND MARGINAL AREAS OF THE PARAGUAY AND PARANA RIVERS
}

\author{
Jorge Alfredo Alberto \\ Supervisor: Ana María Foschiatti \\ Coodirector: María Emilia Pérez
}

- Abstract -

Landscape is the result of processes in continuous interaction in time and space that it is reflected in the uses of the soil. The object of study of this work, is referred to the fringes next to urban centers, that are subjected to constant push of the growth of the citybecause of the residential function and other complementary activities (services and infrastructures) that leads to a dynamic and complex space because the intense urbanization process generates a speculative sector that appropriates land with agricultural rent to obtain urban rent.This "border territory" is subject to economic processes related to the capitalist valorization of space, as a result of actual or potential incorporation of new land to the city.

In the area selected for study, it is observed that the urban growth implies the incorporation of new rural areas into the complex system mutations such as the city and its transition areas, disregarding the relationships between the demands of these proposals, the dynamics of natural conditions and the dynamics of previous traditional activities(agricultural, livestock) All this, complicates even more, the environmental problems arising from the transformation of the space involved.

The growth of this transitionspace of the AMGR presents four basic space forms of urban occupation,they are; continuous development in low density, development in strip,discontinuous development or "leapfrog", and external urbandispersed development. The effect of this phenomenon is the increase in road infrastructure and intra-urban mobility that accentuate the concomitant growth of urban stain with marginalization bags, poverty and urban voids, to which are added, urbanized areas with a high degree of hydrological vulnerability.

This analysis on occupation of the urban over the rural, with special emphasis on the transitional spaces, helped to deduce the behavior of the land situation, verify its organization and the problems that affect this particular area.

Key words: Urban growth, urban-rural transitionspaces, hydro-geomorphological conditions, natural tendency, human action, use of soil, problems, diagnosis and administration.

Publicado en formato digital: Dr. Prof. Jorge Alfredo Alberto. USO DEL SUELO CON FINES URBANOS EN LAS TERRAZAS Y ÁREAS MARGINALES DE LOS RÍOS PARAGUAY - PARANÁ. RESÚMENES DE TESIS. Revista Geográfica Digital. IGUNNE. Facultad de Humanidades. UNNE. Año 14. № 27. Enero - Junio 2017. ISSN 1668-5180 Resistencia, Chaco

En: http://hum.unne.edu.ar/revistas/geoweb/default.htm 
Revista Geográfica Digital. IGUNNE. Facultad de Humanidades. UNNE.

Año 14. № 27. Enero - Junio 2017. ISSN 1668-5180 Resistencia, Chaco

\title{
LAND USE FOR URBAN PURPOSES ON THE TERRACES AND MARGINAL AREAS OF THE PARAGUAY AND PARANA RIVERS
}

\author{
Jorge Alfredo Alberto \\ Supervisor: Ana María Foschiatti \\ Coodirector: María Emilia Pérez \\ - Papers -
}

\section{Introduction}

The present thesis has like goal to study from the Geography some distinctive characteristics of the urban growth of the Metropolitan Area of the Great Resistance, putting special emphasis in the development that takes place in the urban transition spaces - rural of this agglomerate.

In the same one they are kept in mind the phenomenon of the urban expansion associated with the development of the urban stain and the territorial fragmentation, to those that sink the conditions hydro geomorphology and the natural tendency of the place. These elements, together with the dynamics of the system, define the appearance and growth of the city and their behavior gives place to problematic in connection with the degradation of the environments and its population's vulnerability.

In this aspect, the natural tendency together with the processes antrópic acts, on the whole, in the configuration of areas with particular physiognomies. The results of these processes and interactions, be already of natural order or antrópic they configure in the space a mosaic of physiognomies that denominated landscapes.

The notion of "landscape" it contributes the socio-cultural dimension to the problems of ordination of the territory, for what could enrich the proposals of territorial administration with the deepest knowledge in the relationship among the natural, social, economic and cultural processes, facilitating the understanding of the society in general with regarding the transformations caused by these processes.

For it, the methodological mark for the geographical study of the topic in question is based on the system GTP (geosistem, territory, landscape) presented by Claude and Georges Bertrand (1968) that allows to understand each other the processes and characteristic main of the landscape in its geographical dimension

Being been worth of this focus "geographical and systemic" that can mean the starting point of new interpretations, the thesis work it will contribute a rehearsal conformed by an orderly group of graphic and cartographic representations, with their corresponding one poured of concepts.

Publicado en formato digital: Dr. Prof. Jorge Alfredo Alberto. USO DEL SUELO CON FINES URBANOS EN LAS TERRAZAS Y ÁREAS MARGINALES DE LOS RÍOS PARAGUAY - PARANÁ. RESÚMENES DE TESIS. Revista Geográfica Digital. IGUNNE. Facultad de Humanidades. UNNE. Año 14. № 27. Enero - Junio 2017. ISSN 1668-5180 Resistencia, Chaco

En: http://hum.unne.edu.ar/revistas/geoweb/default.htm 


\section{Methods and Technical}

For the development of the work a method was applied for the study of the landscape supported in a geographical conception, following the principles of the geography (localization, causation, connection and evolution) and to center in a systemic vision to conclude in the geographical synthesis.

This focus responded to the necessity of to detect and to characterize the main types of resulting landscapes of natural processes and antrópics in the AMGR and its surroundings, centered in the urban expansion of this metropolises it has more than enough rural spaces; being been worth for it of cartographic bases, of synthesis and historical, supplemented with air pictures and images satellites. These resources were guided to the use of computer tools that they allowed to analyze and to evaluate the different variables that intervene in this process.

\section{The urbanization in the regional context}

The most important aspects in the urban growth of the cities of the NEA during these last years were associated to the impact taken place at the beginning of last decade for the process of privatization of the companies of the state, the deregulation and opening of the economic activities and the initial effects of the stabilization of the economy and the expansion of the credit that it energized and it modernized some commercial activities and of services linked to the financial, energy sectors, of communication and of the construction.

The result on the urban space of these processes brought harnessed, on one hand, the renovation of the urban image of the center of these cities and of some revalued areas, as product of the public expense in infrastructure works and the investment deprived in the commercial sector of services and real estate, while, on the other hand, the expansion of big residential areas of low environmental quality and faulty services as product of the action would self-manage of the popular sectors and the public residence politics that have contributed to define an urban space of contrasts and social fragmentation.

On this process she had an important incidence the urban segregation taken place by the installation of big public works of infrastructure in the main cities of the region that contributed to redefine socially more homogeneous areas toward their interior and shoot among them, in hierarchical terms, I eat likewise, the drop quality of the public space of these areas promoted by the public politicians that have not contributed to the population's social integration.

\section{Condition hydro geomorphology of the place}

The characteristic morphology and topography of the place of the AMGR and next areas are the result of the dynamics of the river Paraná that behaves as a hydrological and topographical great regulator of the space subject to study due to the magnitude of their basin and the flow that it transports, what generates countless fluvial forms (current or abandoned)

Publicado en formato digital: Dr. Prof. Jorge Alfredo Alberto. USO DEL SUELO CON FINES URBANOS EN LAS TERRAZAS Y ÁREAS MARGINALES DE LOS RÍOS PARAGUAY - PARANÁ. RESÚMENES DE TESIS. Revista Geográfica Digital. IGUNNE. Facultad de Humanidades. UNNE. Año 14. № 27. Enero - Junio 2017. ISSN 1668-5180 Resistencia, Chaco

En: http://hum.unne.edu.ar/revistas/geoweb/default.htm 
that impact in the relief generating topographical irregularities. The action antrópic on this natural space, they have been made feel from the beginnings of the occupation, so much for the uses of agricultural floor of the high lands as for the forest exploitation. The expansion of the built area, the services and infrastructures like the layout of communication roads, it was given in all senses, but especially, with an eye toward the connection of the port with the local production areas and with the most distant areas in the territory.

In consequence he modified this way the physiognomy of the area of hydrological influence of the Black river and of their own wandering strip, not alone for the suppression of elements but for the alterations of those that were, phenomenon that repeats at the present time in the riversides of the river Gullet and contained plains, next to Colonia Benítez. This situation of filler of the lagoons, of their fragmentation or of their isolation of the main course, it doesn't only impact in the depression when modifying the primitive morphology, but rather he/she loses temper the hydrological behavior that associates to her.

As it is the case of the AMGR, most of those "environmental problems" they depend on the separation "initials" in the selection or use of the original place. In occasions, the transformation of the space natural initial for intensive uses, overcomes natural conditions generating a certain one "I collapse" of such a place. The transformation of the natural environment for effects of the urbanization, one in the most intensive ways in modification of this environment constitutes, being developed in certain cases, extremely critical and irreversible environmental situations.

\section{The AMGR as organizer of the dynamics of urban development}

The growth of the AMGR presents four recognizable basic space forms of occupation with urban ends that are; I develop I continue in low density, development in strip, discontinuous development or in "Sprawl", and urban external dispersed development. The effect of this phenomenon is the increase of systems of infrastructure routes and of mobility among towns that accentuate the growth of the concomitant urban stain with bags of marginal, urban holes and poverty, to what they sink spaces urbanized with a high grade of hydrological vulnerability.

The planning absence in the urban peripheries is very visible. There the reality is: an extreme environmental degradation, an accented urban functional separation between the outlying colonies and the city, an use of the irrational floor, a dispersion and equipment inadequacy and services, a human mass disintegrated socially that it generates problems of lack of adaptation and it misses of sense of ownership the place in that they live for the absence of identity with the same one.

\section{You condition and dynamic of a ruderal space subject to the pressure of the urban expansion}

Among the most prominent characteristics in the place thatshe understands the area subject to study they can be detailed that it is a complex alluvial plain where the presence of

Publicado en formato digital: Dr. Prof. Jorge Alfredo Alberto. USO DEL SUELO CON FINES URBANOS EN LAS TERRAZAS Y ÁREAS MARGINALES DE LOS RÍOS PARAGUAY - PARANÁ. RESÚMENES DE TESIS. Revista Geográfica Digital. IGUNNE. Facultad de Humanidades. UNNE. Año 14. № 27. Enero - Junio 2017. ISSN 1668-5180 Resistencia, Chaco

En: http://hum.unne.edu.ar/revistas/geoweb/default.htm 
Revista Geográfica Digital. IGUNNE. Facultad de Humanidades. UNNE. Año 14. № 27. Enero - Junio 2017. ISSN 1668-5180 Resistencia, Chaco

high lands of low width is observed that are occupied for woody whose populations are tolerant to the action of the floods and droughts moderately lingering. These they are inserted among low lands whose slow glide takes place in way to laminate with a great development in the territory where the permanency of water is conditioned by the effect of having braked that they generate the topographical and biological obstacles, and that they are accentuated with the antrópics.

In this urban interface - rural studied it is observed that the coexistence of both uses is contradictory, since in the same one its population's coexistence is hindered in relation to interests dissimilar, but the competition is tolerated between the urban development and the rural uses, receiving in consequence population's type: urban population under search of smaller costs of life or better environmental conditions and of life, rural population in search of new work opportunities for the reduction and degradation of their lands, and the installation of activities of services characteristic of the urban life (occupations, you tame, etc.) that respond to the regional and national dynamics from the small one to the great scale in their group.

\section{Dynamics and configuration of the uses of the floor of a ruderal space subject to the pressure of the urban expansion}

That outlined evidence previously the drop integration among the natural and social, since they are not kept simultaneously in mind the diverse types of social actors, the objectives and official goals - sectoral, the incidence that has the effect of external interventions to obtain short term benefits, the development politicians and their quick changes, the distribution of the benefits of the use of the resources, etc.

This problematic ones, in their group, they give place to the loss of economic attractiveness of the primary productions (agricultural, cattleman, horticultural and forest) in the area, generating a transformation in the valuation of the use of the floor, since this she stops to have a speculative value from the productive point of view (agricultural and cattle aptitude, etc.) to acquire a speculative value of real estate type (it distances to the route and populated centers, services, etc.) that derives in the accentuation of urbanization processes starting from mensurations and constructions.

These processes, organized in groups, will impact in the dynamics and the behavior of the evolution of the landscape by means of a logical sequence of changes of properties that you/they would respond, in their group to the action antrópic on the study area that they would be accentuated of not taking the pertinent preventive measures, giving place to problematic even more complex that those diagnosed and analyzed in this work, therefore more difficult of to palliate and to solve. It is observed that the process of urban expansion on the space subject to study:

- it contributes to a degradation of the equipment and public services.

- it diminishes the effective maintenance of the existent infrastructures.

Publicado en formato digital: Dr. Prof. Jorge Alfredo Alberto. USO DEL SUELO CON FINES URBANOS EN LAS TERRAZAS Y ÁREAS MARGINALES DE LOS RÍOS PARAGUAY - PARANÁ. RESÚMENES DE TESIS. Revista Geográfica Digital. IGUNNE. Facultad de Humanidades. UNNE. Año 14. № 27. Enero - Junio 2017. ISSN 1668-5180 Resistencia, Chaco

En: http://hum.unne.edu.ar/revistas/geoweb/default.htm 
Revista Geográfica Digital. IGUNNE. Facultad de Humanidades. UNNE.

Año 14. № 27. Enero - Junio 2017. ISSN 1668-5180 Resistencia, Chaco

- it increases the social costs of transport.

- it consumes more resources than other patterns of urban development.

- it limits to great part from the local population to the access to work positions in the AMGR.

- it increases the expenses in the time of transport.

- it degrades the quality of the air and of the water.

- it supposes an alteration or permanent destruction of the natural habitats.

8. Behavior tendency and wanted of the dynamics of the uses of the floor of a ruderal space subject to the pressure of the urban expansion

As it has been mentioned in remote previous, in the identification of the problems he leaves of an integrated focus and systemic of the landscape, with the pretense of offering limits for the environmental classification that the territorial system should reach.

For statement I outline one worked on two big systems, the natural one and the antrópic; in the first one one kept in mind only physical and biological aspects that characterize the behavior of the study area, without for it to pass for high the human action on the same ones. On the other hand, the system antrópic was organized starting from four dynamic aspects and interrelated to each other: the productive one (forest exploitation, cattleman, agricultural, horticultural and industrial), the real estate one, the population and the infrastructures and services.

In relation to the natural system, as much the physical aspect as the biological one present a strong degradation of the environment, result of the accentuation of natural processes (erosion, to deposit, flood) and the alteration of the original vegetable formations through the action antrópic (dilapidation, exploitation, invaders' advance, extinction)

With regard to the system antrópic, in the productive aspect that implies the primary activities and of transformation, most of the identified problems is related with the degradation of the means for on exploitation, erosion, contamination and illnesses, added to the scarce productive economic profitability of the same one by coordination lack and complementation from the exploitation to level property, to what is added the scarce transformation, preservation, promotion and commercialization of the obtained products.

In the real estate aspect reference is made to the ends of occupation of the floor. In the same one, an alteration of the space qualities is observed by an anarchical occupation, result of an accented real estate speculation that responds, in the first place, to a quick demographic growth and an urban concentration for migratory processes of near rural environments and of the city of Resistance, and in second place, for its vicinity in distance and time to this last one, the one which, in the decades of 8090 she has had a quick growth of the urbanized space, accentuating you this process in these last 15 years.

This problem derives in an it measures of productive, artificial lands the landscape with prairies and forests of endogenous and exogenous species indistinctly, deficiency of services

Publicado en formato digital: Dr. Prof. Jorge Alfredo Alberto. USO DEL SUELO CON FINES URBANOS EN LAS TERRAZAS Y ÁREAS MARGINALES DE LOS RÍOS PARAGUAY - PARANÁ. RESÚMENES DE TESIS. Revista Geográfica Digital. IGUNNE. Facultad de Humanidades. UNNE. Año 14. № 27. Enero - Junio 2017. ISSN 1668-5180 Resistencia, Chaco

En: http://hum.unne.edu.ar/revistas/geoweb/default.htm 
Revista Geográfica Digital. IGUNNE. Facultad de Humanidades. UNNE. Año 14. № 27. Enero - Junio 2017. ISSN 1668-5180 Resistencia, Chaco

and existent infrastructures, situation that is deepened by the absence of a project of planning of urban expansion and for the lack of in agreement fiscal politicians to the use of the floor.

In the demographic aspect the population's quick growth is visualized, the one that concentrates on nuclei located in the places Tres Horqueta, and El Tropezón and in the town of Colonia Benitez. Great part of this it comes from environments rural bordering, where the agricultural activities are in a crisis situation and recession; although in the last years, like it was commented above, a marked population tendency coming from the AMGR is observed in recreation search or with residential ends, I process accompanied by a pendulous movement by labor questions.

In the case of the local population, an important number of unoccupied people is observed with unsatisfied demands of housings and basic service, that which generates marginal establishments, lowers conditions of illegality, in areas with flood risk, what puts in danger the physical integrity of the same ones, situation that deepens the presence of nuclei of poverty, increasing criminal facts and of insecurity, as much in the goods as in people in general.

In relation to second o'clock, the possible increment of the spaces urbanized through housings and, the growing interest in real estate acquisition with a strong mensuration and degradation of productive lands without a plan rector that the east, they notice for a next future the urban character of the area with a density of saturation of very important floor, where the capacity of contention of the services and infrastructures will be overcome, being faulty. This situation, next to the lack of the local actors' coordination, would deepen the real estate speculation altering the space qualities and the form of its residents' life, to what an increment of the confrontation would be added among neighbors by differences of interests and of customs.

In the aspect of infrastructures and services, the observed problems are the result of the population's marked growth and the quick increase of the urbanized surfaces and built, supplemented with processes of occupation of rural spaces with productive ends. It is observed that in rural environments these don't respect the natural tendency in most of the cases, for example the layout of roads and faulty sewer systems that limit the glide of the excess dilute. On the other hand, in urban environments the unsatisfied demand of housings of social plans, the deficiency in the supply of drinkable water, the limited capacity of contention of the educational and sanitary system, is noticed and finally the lack of basic services of sewers, situation that demonstrates a notorious loss in the quality of the residents' life.

A negligence of the cultural and natural patrimonies is also perceived, for isolation and indifference to the interests of the community on the part of the main social actors, or, of population it inserts coming from other areas that are not represented by the same rules or ideals.

Publicado en formato digital: Dr. Prof. Jorge Alfredo Alberto. USO DEL SUELO CON FINES URBANOS EN LAS TERRAZAS Y ÁREAS MARGINALES DE LOS RÍOS PARAGUAY - PARANÁ. RESÚMENES DE TESIS. Revista Geográfica Digital. IGUNNE. Facultad de Humanidades. UNNE. Año 14. № 27. Enero - Junio 2017. ISSN 1668-5180 Resistencia, Chaco

En: http://hum.unne.edu.ar/revistas/geoweb/default.htm 
Definitively the lack of cohesion and coordination, at local level, for the maintenance and amplification of infrastructures and services impact in a negative way in the structural systems of the community equipment, accentuating the crisis in the population's quality life.

Finally, keeping in mind that tried until here, they can intend through trees of objectives possible opportunities to overcome the problems, that is to say starting from the conflicting situations or of detected imbalance a series of conditions or situations are formulated waited in the future that you/they are wanted and realizable in the practice.

In synthesis, all the problems identified summary in four key problems that are detailed next:

1. degradation of the original natural means in their entirety.

2. inefficient productive systems in relation to profitability and sustainable.

3. alteration of the space qualities.

4. loss of the quality of life.

Starting from the key problems, they have been defined the following objectives guided to their solution:

1. to palliate and/or to diminish the degradation of the natural means.

2. to foment initiatives to improve the efficiency of the productive systems.

3. to diminish the alteration of the space qualities.

4. to palliate or to diminish the loss of the quality of life.

\section{Conclusion}

In the area selected for their study, it is observed that the urban growth implies the incorporation of new rural territories in the complex system that he understands a space in mutation like it is it the city and its transition areas, without keeping in mind for it the existent relationships among the demands that incorporate these proposals and the dynamics of the natural conditions together with the dynamics characteristic of the previous traditional activities to this process (agricultural, cattlemen), that which complex the derived environmental problems of the transformations of the involved space.

The growth of this space of transition of the AMGR presents four recognizable basic space forms of occupation with urban ends that are; I develop I continue in low density, development in strip, discontinuous development or in "sprawl", and development dispersed urban external border:

- The continuous development in low density, is that of more territory consumption in the margins of the metropolitan area, transforming it in urban floor. This expansion type is based on the extension of the nets of urban infrastructure as being water, electricity, etc. (Villa Fabiana, Villa Camila, Monte Alto, La California, , etc.)

Publicado en formato digital: Dr. Prof. Jorge Alfredo Alberto. USO DEL SUELO CON FINES URBANOS EN LAS TERRAZAS Y ÁREAS MARGINALES DE LOS RÍOS PARAGUAY - PARANÁ. RESÚMENES DE TESIS. Revista Geográfica Digital. IGUNNE. Facultad de Humanidades. UNNE. Año 14. № 27. Enero - Junio 2017. ISSN 1668-5180 Resistencia, Chaco

En: http://hum.unne.edu.ar/revistas/geoweb/default.htm 
Revista Geográfica Digital. IGUNNE. Facultad de Humanidades. UNNE. Año 14. № 27. Enero - Junio 2017. ISSN 1668-5180 Resistencia, Chaco

- The development in strip is given in the line of the big corridors of transport that they leave the central areas of the city (especially the route National $n^{\circ} 11$ and the Railcar Nicolás Hazel grove) The adjacent areas to the corridor are developed with urban uses, and the areas far to the same one maintain its rural character. With the time this rural earth becomes to urban, in the measure that traverse corridors of transport or local roads arise.

- The discontinuous development or in "Sprawl", they are discontinuous urbanizations of the margins of the metropolitan area or of conurbations in strip, as the case of the next urbanizations to the town of Colonia Benítez (Las Marías, Chacras de Benítez, etc.) This development type is the one that requires of more investment in urban services to be able to be materialized.

- The urban dispersed development associates to the expansion concept in dividing type housing of lands, in natural environments (agricultural, forest, etc.), beyond the suburbs of the city. This establishment pattern has also been denominated "I develop in extended low density", and it differs significantly of the urban patterns, of suburbs or rural. The same ones are in an accented development in the itinerary understood between Margarita Belen and Colonia Benítez to the route National $n^{\circ} 11$ and the old road among these two towns known as "The diagonal."

The resulting product of the four above urbanization processes causes evident impacts as much in the AMGR as in the area selected for such study as: the loss of population of the consolidated urban spaces that it generates for the administrations of the city decrease and escape in the perception of taxes; the increase in the expenses of the infrastructure of roads difficult to finance with the drops densities; the consumption of lands periurban dedicated to the production, the little use of the urban center, to what sinks the residential segregation in relation to the differentiation as for the types of services (you buy, entertainments, education, health)

In this "perimeter", in constant increase, they cohabit all the commented possibilities that they result in the demands of very disparate, complex and difficult requirements of satisfying, giving place to a variety of problematic social, legal, economic and in their group, environmental. As answer to the above situation, the human groups should appeal to the outlying spaces and urban holes that can be in risk because these territories, far from gathering qualities of habitability, are vulnerable for their physical conditions - natural.

This analysis approach on the occupation of the urban thing on the rural thing, with special emphasis in the transition spaces, allowed to deduce the behavior of the situation of the earth, to verify the organization of the same one and the problems affect to the area in question. Keeping in mind the above aspects that impact on the study area the necessity arises of to deepen and to insist in the future action lines as:

Publicado en formato digital: Dr. Prof. Jorge Alfredo Alberto. USO DEL SUELO CON FINES URBANOS EN LAS TERRAZAS Y ÁREAS MARGINALES DE LOS RÍOS PARAGUAY - PARANÁ. RESÚMENES DE TESIS. Revista Geográfica Digital. IGUNNE. Facultad de Humanidades. UNNE. Año 14. № 27. Enero - Junio 2017. ISSN 1668-5180 Resistencia, Chaco

En: http://hum.unne.edu.ar/revistas/geoweb/default.htm 
Revista Geográfica Digital. IGUNNE. Facultad de Humanidades. UNNE.

Año 14. № 27. Enero - Junio 2017. ISSN 1668-5180 Resistencia, Chaco

- To study the technologies possible to apply without loss of work positions for expulsion.

- To develop novel, alternative, complementary and diversified productive systems that they exploit the local resources in a sustainable way.

- To develop novel, alternative, complementary and diversified productive systems that they exploit the local resources in a sustainable way.

- To define and to develop critical centers of local economic development, putting the effort in identifying urbanization nuclei that present a potential development in support of the agricultural activities.

- To work in the increment of the commercial exchange among near populated centers so that they become, also, in centers of distribution of the agrarian products.

- To palliate the insufficient access or the bad distribution of lands, either for political, social, real estate or economic obstacles.

- To legislate to not limit the usurpation of lands by means of a system subject to notarizing usual of the private property, for it will be determined it the property of the parcels and to register the right of the individuals on the lands, starting from norms that are accessible to the groups of low revenues.

- To propose energetic measures to limit the urban expansion in the fertile agricultural areas, conserving them for the production of foods and I eat spaces free of contamination.

- To build the structure that you guide the change of the urban activity and of the rural activity, working in favor of educating in an in agreement culture with the sustainable development.

\section{Bibliography bases consulted}

1. Alberto, Jorge A. (2005) "Problemáticas ambientales resultantes de la ocupación urbana en ambientes fluviolacustres. Estudio de caso: Área Metropolitana del Gran Resistencia (AMGR)”. En Revista Geográfica no 138. Instituto Panamericano de Geografía e Historia (IPGH) Mexico DF. Julio - diciembre 2005. pp. 109 - 127

2. Alberto, Jorge A. (2007) "Vulnerabilidad Ecológica y natural". En: Foschiatti, Ana Maria (Eds.) "Aportes conceptuales y empiricos de la vulnerabilidd global”. Resistencia. EUDENE. UNNE. Resistencia. Argentina. pp. 117 - 158.

3. Barnes, K., Morgan, J. (2002) "Sprawl development: its patterns, consequences, and measurement" Center for Geographic Information Sciences, Towson University. [en línea] Junio 2010: http://pages.towson.edu/morgan/files/Sprawl_Development.pdf

Publicado en formato digital: Dr. Prof. Jorge Alfredo Alberto. USO DEL SUELO CON FINES URBANOS EN LAS TERRAZAS Y ÁREAS MARGINALES DE LOS RÍOS PARAGUAY - PARANÁ. RESÚMENES DE TESIS. Revista Geográfica Digital. IGUNNE. Facultad de Humanidades. UNNE. Año 14. № 27. Enero - Junio 2017. ISSN 1668-5180 Resistencia, Chaco

En: http://hum.unne.edu.ar/revistas/geoweb/default.htm 
Revista Geográfica Digital. IGUNNE. Facultad de Humanidades. UNNE. Año 14. № 27. Enero - Junio 2017. ISSN 1668-5180 Resistencia, Chaco

4. Barreto, M. A. y Ebel, G. A. (2013) "Crecimiento, distribución y composición de la problacion urbana y rural en el Chaco entre 1991 y 2010". Revista ADNea n 1. Vol. 1. Diciembre 2013, Facultad de Arquitectura y Urbanismo. UNNE. pp. 77 - 88.

5. Bertrand, G. (1968) "Paysage et geographie physique globale: esquisse méthodologique". Rev.Géograph. Pyrénées et du Sud-Ouest, 39(3):249-272, Toulouse.

6. Bolòs, I.; Capdevila, M. (1992) "Manual de Ciencia del Paisaje. Teoría, métodos y aplicaciones". Masson, Barcelona. 252 pp.

7. Bryant, C y Russwurn, L. Fuente: Bryant, C. R. ;Russwurn, L. H.,McLellan, A. G. (1982) "The city's countryside. Land and its management in the rural-urban fringe". Longman.- New York.- $249 \mathrm{pp}$.

8. Dollfus, O. (1976) "El espacio geográfico". Colección ¿Qué se? № 111, Ed. Oikos-Tau, Vilassar de Mar.

9. Foschiatti, A. M. (Eds.) (2008) "Aportes conceptuales y empiricos de la vulnerabilidd global". Resistencia. EUDENE. UNNE. Resistencia. Argentina. pp. 425.

10. García, R. (2006) "Sistemas complejos: conceptos, método y fundamentación epistemológica de la investigación interdisciplinaria". Editorial Gedisa. Barcelona.

11. Gaviria, M. (1985) "La competencia rural-urbana por el uso de la tierra", Agricultura y Sociedad, pp. 245-261

12. Ibarra Benlloch, P. (1993) "Una propuesta metodológica para el estudio del paisaje integrado". En revista Geographicalia 30. Revista del Departamento de Geografía y Ordenación del Territorio. Universidad de Zaragoza. Diciembre 1993. pp. 229-242

13. Mignone, A. M. (2004) "El proceso de urbanización en el Gran Resistencia, a principios del siglo XXI. El caso de los asentamientos espontáneos". Actas VI Encuentro Internacional Humboldt. Villa Carlos Paz., Córdoba. 13 a 17 de septiembre de 2004.

14. Pierre George (1985) "La acción del hombre y el medio geográfico". Traducción de Alexandre Montserrant. Ediciones Península, Historia, Ciencia, Sociedad no 61. Barcelona. España.

15. Pilar Serra, Y. (2003) "Resistencia: geomorfología del sitio, expansión urbana y afectación hídrica". XIII Encuentro Nacional de Profesores de Geografía. Agosto 2003.

16. Popolizio, E. (1982) "La geomorfología en los estudios ecológicos de la llanura". En revista. Geociencias XII. Publicación del Centro de Geociencias Aplicadas. Universidad Nacional del Nordeste. Resistencia, Chaco, Argentina.

17. Popolizio, E. (1989) "Algunos elementos geomorfológicos condicionantes de la organización espacial y las actividades del NEA". En revista Geociencias no XVII. Centro de Geociencias Aplicadas, UNNE. Resistencia.

\footnotetext{
Publicado en formato digital: Dr. Prof. Jorge Alfredo Alberto. USO DEL SUELO CON FINES URBANOS EN LAS TERRAZAS Y ÁREAS MARGINALES DE LOS RÍOS PARAGUAY - PARANÁ. RESÚMENES DE TESIS. Revista Geográfica Digital. IGUNNE. Facultad de Humanidades. UNNE. Año 14. № 27. Enero - Junio 2017. ISSN 1668-5180 Resistencia, Chaco

En: http://hum.unne.edu.ar/revistas/geoweb/default.htm
} 